\title{
Novel Developments in Environmental Transmission Electron Microscopy
}

\author{
P.A. Crozier \\ School of Mechanical, Aerospace, Chemical and Materials Engineering, Arizona State \\ University, Tempe, AZ, 85287-6106
}

Environmental transmission electron microscopy (ETEM) is an important tool for in situ characterization because reactive gases may have a strong influence on the structure and composition of materials. The ability to study materials at elevated temperatures under reactive gases makes it possible to investigate the atomic scale changes taking place during materials synthesis and in materials under reactor conditions. For example, by correlating catalytic reactor data with suitably designed ETEM observations, it is possible to map out structure-property relations in catalysts. In ETEM, the gas is mostly confined to the region around the TEM sample and not allowed access to the rest of the column or the electron gun. This is usually accomplished using either windowed cell holders or a special differential pumping system within the TEM. The former allows high pressures (up to an atmosphere or higher) to be achieved while the latter provides only intermediate pressure (up to 20 Torr) but avoids the superposition of the window signal onto images, diffraction patterns or spectra. Heating can be performed up to $1000^{\circ} \mathrm{C}$ or higher although the thermal conductivity and gas pressure can make precise temperature determination more challenging for some configurations. The addition of aberration correction makes the modern ETEM a powerful tool for studying gas-solid interactions at resolutions below $1 \AA$. We are currently extending the capability of ETEM by developing operando techniques and introducing variable wavelength light illumination.

In heterogeneous catalysis, we really want to correlate the structural and chemical changes taking place in a catalyst with simultaneous measurement of gas reaction products. Techniques where catalysis is simultaneously measured with in situ characterization of the catalyst have been developed in other areas and are called operando methods [1]. While such gas analysis may be performed with online mass spectrometers, we have developed a method of performing gas analysis in the ETEM using in situ electron energy-loss spectroscopy (EELS) [2]. This approach allows real time analysis of the volume of gas adjacent to the TEM sample and can be performed rapidly with typical acquisition times being a few seconds (or less for low-loss spectroscopy). Quantification can be performed either with the core-loss spectra or with the low-loss spectra. Figure 1 shows at typical spectrum from a $\mathrm{CH}_{4}$ and $\mathrm{O}_{2}$ gas mixture recorded at a pressure of 2 Torr in our FEI Tecnai F20 ETEM. We can measure the molar concentration of $\mathrm{O}_{2} / \mathrm{CH}_{4}$ by fitting a linear combination of the spectra from $\mathrm{O}_{2}$ and $\mathrm{CH}_{4}$. We are currently employing this approach to detect catalytic products in the microscope so that we can correlate the structural transformation with the onset of catalysis [3]. This will essentially open up a new field called operando TEM for catalysis research.

Motivated by a desire to improve solar energy conversions, we are also incorporating a variable wavelength high-brightness light source into our FEI Tecnai F20 ETEM. Solar fuel generation allows solar energy to be directly coupled into a fuel molecule like methane which then serves as the energy storage medium. These approaches involve some form of artificial photosynthesis for water splitting and/or reduction of $\mathrm{CO}_{2}$. The current photocatalysts have very low efficiencies or stabilities and it is critical to investigate the surface and bulk phase transformations that take 
place in the presence of reactive gases during photon irradiation. In our design (see schematic figure 2), we employ an Energetiq laser driven light source and couple light into the reaction cell of the microscope using a quartz fiber. The fiber is kept some distance from the TEM sample holder so that heating in reactive gas environments can be performed without damage to the fiber [4]. We plan to investigate the phase changes taking place on the surface of the catalyst as a function of wavelength, photon intensity, temperature and gas.

\section{References:}

[1] Bañares, M. A., and Wachs, I. E., (2002), Journal of Raman Spectroscopy, 33, 359.

[2] Crozer, P.A. and Chenna, S. (2011), Ultramicroscopy, 111, 177-185.

[3] Chenna, S. and Crozier, P.A. (these proceedings)

[4] Miller, B.K. and Crozier, P.A. (these proceedings)

[5] The support from the National Science Foundation (NSF-CBET-0553445), US Department of Energy (DE-SC0004954) and the use of TEMs at the John M. Cowley Center for High Resolution Microscopy at Arizona State University are gratefully acknowledged.

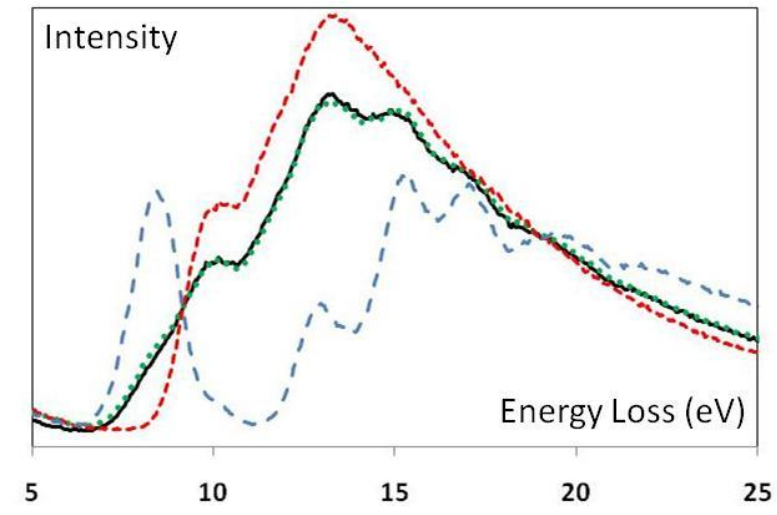

FIG 1: Low-loss spectra from gas mixture of $2 \mathrm{CH}_{4}+\mathrm{O}_{2}$ (black solid line) at about 2.6 Torr plus low loss spectra from $\mathrm{CH}_{4}$ (red dotted) and $\mathrm{O}_{2}$ (blue dashed). The simulated spectrum (green dotted) is a linear combination of the spectra from $\mathrm{CH}_{4}$ and $\mathrm{O}_{2}$ and shows the $\mathrm{O}_{2} / \mathrm{CH}_{4}$ molar ratio to be 0.6.

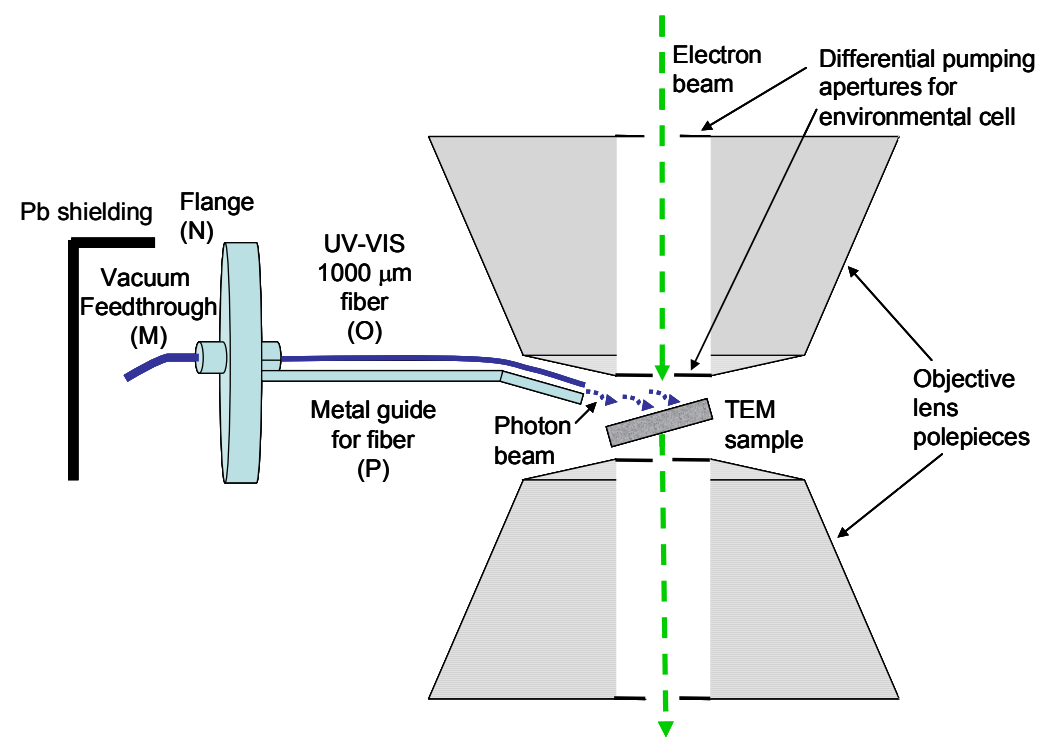

FIG 2: Schematic of the light setup into the in-situ environmental TEM showing the upper and lower pole pieces of the electron microscope with the optical fiber allowing photon irradiation of a heated sample in a reactive gas environment. 